\title{
Estresse emocional entre cuidadores informais de pacientes em cuidados paliativos
}

Emotional stress between informal caregivers of patients in palliative care

Estrés emocional entre los cuidadores informales de pacientes en cuidados paliativos

\author{
Elayne Mágda Andrade do Nascimento' $\odot$; Mariana de Sousa Dantas Rodrigues' $\odot$; Carla Braz Evangelista' $\odot ;$ \\ Ronny Anderson de Oliveira Cruz' ๑; Alana Vieira Lordão" ๑; Patrícia Serpa de Souza Batista" @
}

'Centro Universitário de João Pessoa, João Pessoa, Paraíba, Brasil; "Universidade Federal da Paraíba, João Pessoa, Paraíba, Brasil

\section{RESUMO}

Objetivo: analisar o estresse emocional de cuidadores informais de pacientes em Cuidados Paliativos. Método: pesquisa de campo, exploratória, com abordagem qualitativa, realizada com 10 cuidadores informais de um hospital filantrópico em João Pessoa, Paraíba, Brasil. Os dados foram coletados entre abril e maio de 2021, mediante questionário online e entrevista semiestruturada virtual, e então, submetidos à análise de conteúdo temática, após aprovação do Comitê de Ética em Pesquisa com Seres Humanos. Resultados: foram obtidas as categorias temáticas: Cansaço físico e emocional do cuidador informal; Cuidado como forma de dever e retribuição do afeto; O cuidado e a necessidade de rede de apoio social e da espiritualidade; Dificuldades na adaptação do cuidador aos Cuidados Paliativos na instituição hospitalar. Considerações finais: é necessária a divisão das responsabilidades do cuidado entre cuidadores informais e outros familiares. Salienta-se a importância da equipe de saúde estimular a dimensão espiritual e social do cuidador.

Descritores: Saúde Mental; Cuidados Paliativos; Cuidadores; Estresse Psicológico.

\section{ABSTRACT}

Objective: to analyze emotional stress among informal caregivers of patients in palliative care. Method: this exploratory, qualitative field study was conducted with 10 informal caregivers from a philanthropic hospital in João Pessoa, Paraíba, Brazil. Data were collected between April and May 2021, through online questionnaire and virtual semi-structured interview, and then submitted to thematic content analysis, after approval by the human research ethics committee. Results: the thematic categories obtained were: physical and emotional fatigue in the informal caregiver; care as a form of duty and retribution for affection; care and the need for a social support network and spirituality; and caregivers' difficulties in adapting to palliative care in the hospital. Final considerations: care responsibilities must be divided among informal and family caregivers. Also stressed is the importance of the health team's stimulating caregivers spiritually and socially.

Descriptors: Mental Health; Palliative Care; Caregivers; Emotional, Psychological.

\section{RESUMEN}

Objetivo: analizar el estrés emocional de cuidadores informales de pacientes en Cuidados Paliativos. Método: investigación de campo, exploratoria, con enfoque cualitativo, realizada junto a 10 cuidadores informales de un hospital filantrópico en João Pessoa, Paraíba, Brasil. Los datos se recogieron entre abril y mayo de 2021, mediante un cuestionario online y una entrevista semiestructurada virtual, y luego se sometieron a un análisis de contenido temático, tras la aprobación del Comité de Ética en Investigación con Seres Humanos. Resultados: Se obtuvieron las siguientes categorías temáticas: Fatiga física y emocional del cuidador informal; Cuidado como forma de deber y retribución del afecto; El cuidado y la necesidad de red de apoyo social y espiritualidad; Dificultades de adaptación del cuidador a los Cuidados Paliativos en la institución hospitalaria. Consideraciones finales: es necesario repartir las responsabilidades de los cuidados entre los cuidadores informales y otros miembros de la familia. Así mismo, se destaca la importancia de que el equipo de salud estimule la dimensión espiritual y social del cuidador. Descriptores: Salud Mental; Cuidados Paliativos; Cuidadores; Estrés Psicológico.

\section{INTRODUÇÃO}

Os Cuidados Paliativos (CP) constituem-se como uma abordagem de cuidados holísticos ativos aos indivíduos, em todas as faixas etárias, que possuam algum sofrimento relacionado à saúde, devido a doenças ameaçadoras da vida. Seu objetivo é prevenir e aliviar o sofrimento e melhorar a qualidade de vida de pacientes com doenças potencialmente fatais, familiares e cuidadores ${ }^{1,2}$, mediante a atuação de equipe interdisciplinar, com formação especializada, e ênfase numa comunicação compassiva, atenção à família e conhecimento no manejo de sintomas ${ }^{3}$.

Tendo em vista a ampla abordagem dos aspectos inerentes ao paciente, inclui-se nessa rede de assistência paliativa o cuidador informal, considerado um elemento fundamental nesse processo ${ }^{4}$. Este cuidador informal caracteriza-se pelo vínculo preexistente com o ser cuidado $^{5}$, e apresenta dois aspectos essenciais, como, não possuir relação de trabalho remunerado e nem formação específica na área de prestação de cuidados ${ }^{6}$. Além disso, é comum uma só pessoa, de forma auto-atribuída, assumir esse papel.

Autora correspondente: Elayne Mágda Andrade do Nascimento. E-mail: elayne.magdaa@gmail.com Editora Científica: Cristiane Helena Gallasch; Editor Associado: Sergio Corrêa Marques 
Tornar-se um cuidador pode ser um processo de início lento ou abrupto, estabelecendo-se desde o diagnóstico de determinada doença, cujo tratamento e evolução desencadeiam um quadro de dependência ${ }^{8}$. A demanda de responsabilidades de cuidado evidencia a sobrecarga acentuada em cuidadores informais, expressa pelos sinais de estresse envolvidos nas situações de mudança e adaptação de rotina, privação de liberdade e lazer, além da realização de cuidados sem técnica, conhecimento ou orientação ${ }^{9}$.

Dentre as novas vivências dos cuidadores, por muitas vezes previamente desconhecidas por eles, encontra-se a administração de medicamentos, higienização no leito, preparo da alimentação, manuseio de drenos, sondas e realização de curativos. Além de lidar com o avanço dos sintomas e a possibilidade de morte iminente do familiar ${ }^{10}$.

Alguns distúrbios psicossomáticos estão relacionados ao estresse emocional, caracterizados por sintomas como cansaço mental, baixa concentração, falha da memória, apatia e indiferença emocional, os quais também são comuns em condições de ansiedade ou depressão, devendo o profissional de saúde estar atento às alterações emocionais do cuidador ${ }^{11}$.

Sob esses aspectos, estudos nacionais e internacionais evidenciam que estes cuidadores se tornam mais vulneráveis a eventos estressores devido a interação e as atividades que sua função requer, principalmente diante de doentes crônicos ${ }^{11-15}$, o que torna necessário a investigação da ocorrência do estresse emocional em cuidadores informais no contexto dos CP.

Além disso, estudos sobre o tema poderão contribuir para o planejamento e implementação de estratégias de prevenção, autocuidado e identificação do fenômeno, repercutindo em efeitos benevolentes na qualidade de vida e na prevenção de potencial adoecimento mental por esse grupo. Dessa forma, realizou-se a presente pesquisa que apresenta a seguinte questão norteadora: quais os fatores associados à ocorrência do estresse emocional de cuidadores informais de pacientes em Cuidados Paliativos?

Para responder ao questionamento, a pesquisa teve por objetivo analisar o estresse emocional de cuidadores informais de pacientes em CP; e identificar os fatores relacionados ao estresse emocional de cuidadores informais de pacientes em CP.

\section{MÉTODO}

Foi realizada uma pesquisa de campo, exploratória, com abordagem qualitativa, na qual foram considerados os critérios do Consolidated Criteria for Reporting Qualitative Research (COREQ) ${ }^{16}$. A coleta de dados ocorreu em ambiente virtual com cuidadores informais das Unidades de Cuidados Paliativos de um hospital filantrópico localizado em João Pessoa, Paraíba, Brasil, durante o período de abril a maio de 2021.

Foram incluídos no estudo os cuidadores informais de pacientes em CP: entre 18 e 65 anos de idade; sem remuneração; que acompanhavam o paciente por um período igual ou maior há seis meses; que possuíam dispositivo digital com rede de internet disponível. Foram excluídos do estudo aqueles cuidadores que estavam no local do estudo apenas para troca de horários de acompanhamento; e que expressaram dificuldade para utilizar o dispositivo digital.

$\mathrm{Na}$ primeira etapa deu-se a aplicação de um questionário online realizado por meio de um formulário na plataforma Google forms $^{\circledR}$, para obtenção de dados sociodemográficos dos participantes.

A segunda etapa correspondeu a uma entrevista semiestruturada realizada por meio de aplicativo de chamada audiovisual, a fim de investigar o estresse emocional no cuidador informal a partir das seguintes questões subjetivas: Como você se sente realizando os cuidados com o paciente? Você acha que consegue realizar a maioria das coisas que você necessita? Como você se organiza e faz planos para o futuro? Você sente que o que faz pelo paciente é suficiente? Quais as dificuldades que você enfrenta nessa relação de cuidado? Você se sente sobrecarregado por causa dos cuidados com o paciente? Você acha que sua saúde foi prejudicada? Se sim, o que faz para melhorar? Você gostaria de encontrar uma saída para essa situação? Você se sente valorizado pelos familiares do paciente? Como você se sentiria se outra pessoa dividisse os cuidados com você?

Os candidatos foram abordados pessoalmente através de visita ao local para apresentação da pesquisadora e do Termo de Consentimento Livre e Esclarecido (TCLE). Após este momento, foi realizado o agendamento do dia e horário disponível pelo cuidador para coleta virtual, que teve duração máxima de 30 minutos, bem como a recomendação de local adequado e privativo para a condução do estudo. Os áudios da entrevista foram captados através de um gravador de áudio, e posteriormente, transcritos na íntegra.

Ao todo, foram acessados 15 participantes, no entanto, cinco deles não entraram para amostra por razão de desistência. Dessa forma, o tamanho da amostra foi estabelecido em dez cuidadores informais de pacientes internos no local do estudo. Utilizou-se a amostra não probabilística por conveniência, em que foram selecionados membros da população mais acessíveis, buscando-se a qualidade das ações e das interações em todo o decorrer do processo ${ }^{17}$. 
Os dados foram analisados a partir da metodologia de análise de conteúdo temática a partir das seguintes fases: pré-análise, codificação, categorização e inferência ${ }^{18}$. Na fase de pré-análise, ocorreu a leitura flutuante dos dados empíricos previamente transcritos com a sistematização das ideias iniciais. A codificação desses dados deu-se pela agregação das unidades de registro em cores, seguido da fase de categorização. Por fim, foi realizada a análise e discussão dessas categorias em fase de inferência.

O estudo foi aprovado pelo Comitê de Ética em Pesquisa com Seres Humanos, em conformidade com os preceitos da Lei Geral de Proteção de Dados Pessoais (LGPD) no $13.709 / 18^{19}$ e medidas de proteção, segurança e garantia de direitos dos participantes em ambiente virtual ${ }^{20}$. Uma vez assinado o TCLE por todos os participantes e a fim de garantir o anonimato deles, foram utilizados nomes de espécies de borboletas remetendo à simbologia dos Cuidados Paliativos, dentre eles estão: Ascia monuste (B1), Caligo (B2), Danaus (B3), Dryas julia (B4), Hamadryas (B5), Methona (B6), Morpho (B7), Panacea prola (B8), Phoebis (B9) e Siproeta stelenes (B10).

\section{RESULTADOS E DISCUSSÃo}

Dos participantes da pesquisa, a maioria eram do sexo feminino, fator socialmente reconhecido como construção histórica da figura feminina intrínseca às práticas do cuidar, como observa-se também em alguns estudos ${ }^{3,21}$, e a faixa etária variou entre 18 e 55 anos de idade. A maioria eram solteiros, casados ou divorciados, sendo $30 \%$ cada um deles. Oito deles possuíam filhos (80\%), sete (70\%) se autodeclararam como pardo(a) e seis (60\%) tinham ensino médio completo. Em relação aos antecedentes clínicos pessoais, encontrou-se principalmente a presença de distúrbios osteomusculares $(33,3 \%)$, ansiedade $(27,8 \%)$ e distúrbios do sono $(11,1 \%)$.

Seis $(60 \%)$ participantes relataram não possuir vínculo empregatício, estando suscetível a perder sua autonomia, contrair dívidas, inclusive, perder o apoio social ${ }^{22}$. As ocupações variaram, sendo dois (20\%) dos participantes agricultores. $\mathrm{O}$ grau de parentesco entre os cuidadores e os doentes caracterizou-se em sua maioria como filhos(as) (30\%) e netos(as) (30\%). Nenhum deles recebia remuneração pelo cuidado, seis deles (60\%) predominaram o tempo de cuidado entre seis meses e um ano, enquanto os demais, distribuíram-se por um período variante e superior a um ano.

A partir da análise dos dados, foram identificadas quatro categorias temáticas: Cansaço físico e emocional do cuidador informal; Cuidado como forma de dever e retribuição do afeto; O cuidado e a necessidade de rede de apoio social e da espiritualidade; Dificuldades na adaptação do cuidador aos Cuidados Paliativos na instituição hospitalar.

\section{Cansaço físico e emocional do cuidador informal}

A partir dos discursos dos participantes, verificou-se sinais e sintomas físicos e emocionais, como, problemas de sono e repouso, alimentação, sinais de rebaixamento do sistema imunológico, ansiedade, exaustão emocional, desejo de fuga e irritabilidade como fatores relacionados com o cansaço tanto físico quanto emocional, caracterizados pela alta demanda em relação à rotina diária de cuidados, citados nos trechos a seguir:

[...] Eu não durmo à noite porque ele (padrasto) tem câncer na próstata então fica fazendo xixi de dez em dez min, isso deixa o corpo bem cansado mesmo [...] a impressão que dá é que estou doente, mas, é apenas o cansaço de não dormir direito. (B2)

Me sinto muito sobrecarregada viu... eu fico oito dias direto lá (hospital), aí depois venho em casa e passo só dois ou três dias. (B3)

É uma situação difícil, a gente sabe que é uma situação difícil que ele (pai) tá passando, e se torna um pouco cansativo por conta do estresse do trabalho e tudo mais. (B5)

Minha saúde emocional com certeza foi prejudicada, eu me sinto mais nervosa, uma coceira na pele, de vez em quando dá um frio, um nervosismo... (B6)

[...] Eu comecei a sentir insônia, nos dias no hospital também, chega um determinado horário que eu já estou tão esgotada que às vezes dá vontade de sair correndo do hospital [...] A sensação que eu tenho é que eu vou ter uma crise de ansiedade, [...] esse desgaste realmente está me afetando, sinto que a minha imunidade está baixando, porque começa a aparecer aftas na boca [...] (B7)

Com certeza, me sinto prejudicada fisicamente, por não conseguir me alimentar direito, nem dormir, $e$ também emocionalmente, por parar a minha vida né... (B8)

Foi verificado em estudo que o estresse emocional se relaciona com a sobrecarga, apresentando-se como fator de risco para o desconforto emocional ${ }^{23}$. Os discursos também evidenciaram que o cuidador demonstra vivenciar sofrimento ao perceber que o paciente em CP continua sem apresentar melhora significativa. No entanto, quando o cuidador percebe o processo de atenuação do sofrimento do paciente devido ao controle dos sintomas, este processo de sofrimento multidimensional torna-se menos doloroso para ambos ${ }^{21}$.

Eu queria muito fazer alguma coisa para fazer ele crescer, para melhorar, mas ele é paralisado, parou no tempo... (B4) 
Eu fico em dúvida se é suficiente o que faço por ele por ele não falar, eu queria que ele falasse, para eu ter certeza do que estou fazendo né. (B6)

[...] O sofrimento dela é tão grande que não adianta eu falar pra ela que ela não pode comer nem beber água, mas ao mesmo tempo eu não consigo controlar também o meu sofrimento de desespero. [...] é um sofrimento para ela e é um sofrimento para a gente também. (B7)

Minha maior dificuldade é ver ele naquela situação [...]. (B9)

[...] Não adianta só eu querer que ela viva, ela também precisa querer viver [...]-quando a gente tá conversando ela diz "eu vou ficar boa viu, vamos pra casa logo". Eu acompanho o mesmo pensamento dela, de querer viver, ver ela esperançosa também me dá esperança. (B10)

Sendo assim, é possível analisar que os sentimentos vividos pelo doente estão diretamente ligados com os dos cuidadores, permitindo uma conexão intensa, interpessoal e afetiva entre os envolvidos, mostrando-se atuante para o processo de oscilação emocional do cuidador no contexto de CP, mediado pelos sentimentos do ser cuidado que podem apresentar-se tanto positivos quanto negativos.

\section{Cuidado como forma de dever e retribuição do afeto}

Ao serem questionados acerca do sentimento que possuem ao executar os cuidados com o seu familiar em CP, apesar do cansaço físico e emocional, nove deles expressaram-se de modo positivo. $O$ cuidador familiar detém do compartilhamento de afetos, sentimentos de confiança, conforto e satisfação da necessidade do outro, que expressam sentimentos de satisfação pessoal e gratidão por assumir o papel de cuidador com atenção voltada para o familiar doente receptor de cuidados com sentimento de devoção, amor e afetos ${ }^{12,24-26}$. Neste contexto, foi possível destacar os seguintes trechos:

Eu me sinto bem cuidando dele, porque eu gosto muito dele. [...] a minha vida toda eu devo primeiramente a Deus e depois a ele, foi ele quem me criou, ele me educou, e hoje eu sou a pessoa que sou por ele, então eu faço a minha parte. (B1)

Eu tento dar o melhor de mim né, porque ele está precisando dos meus cuidados né, eu tento estar ali dando o melhor de mim. (B3)

Eu me sinto muito bem cuidando dele, eu estou fazendo o bem ao próximo, eu faria a qualquer ser humano que esteja precisando. (B4)

É satisfatório porque é meu pai né, quanto antes ele sair da situação melhor. (B5)

Me sinto bem em cuidar dele, por ser alguém do meu sangue, que faz parte da minha família [...] eu sinto prazer em cuidar dele. Se eu pudesse dar a melhora dele eu dava. (B6)

Eu sinto que estou fazendo a minha parte, que estou dando o amor que a minha avó precisa nesse momento, os cuidados que ela precisa, eu estou do lado dela no momento mais difícil da vida dela. (B7)

No futuro eu me imagino cuidando dele, ele morando na minha casa. Eu batalhando para cuidar dele e buscar o meu futuro pessoal também. (B8)

[...] É o meu pai né, eu tenho que fazer isso por ele. Eu não gosto de estar em hospital, só que a pessoa tem uma obrigação de ir e a pessoa vai, né isso? [...] então, eu tenho que cuidar dele e tô cuidando. (B9)

Eu me sinto bem, é o cuidado que devemos ter com os pais, com as mães da gente. É coisa dos filhos mesmo cuidar dos pais. É cansativo, mas é à mãe da gente [...] se a gente não cuidar da mãe da gente, quem vai cuidar? ela me deu a vida, se fosse eu quem estivesse doente, ela faria a mesma coisa, estaria do meu lado. (B10)

Geralmente o cuidado é determinado por relações de afeto e compromisso que unem o familiar, amigos ou vizinhos à pessoa dependente, denominando de cuidador informal ${ }^{24}$. Todavia, embora a prática do cuidar envolva sentimentos de satisfação, realização pessoal e orgulho, é possível observar a presença de desgaste emocional, estresse e até problemas de saúde em decorrência desse serviço em ambiente hospitalar e até mesmo no contexto dos $\mathrm{CP}^{8}$.

É importante ressaltar que essa compatibilidade se torna temerosa para o cuidador no que se refere ao risco de negligenciamento de sua própria saúde, a fim de dedicar-se somente ao doente. Tais questões poderão abalar suas dimensões emocionais, além de gerar modificações e privações de atividades pessoais de estilo de vida para incluir as necessidades de seu familiar doente ${ }^{27}$

\section{O cuidado e a necessidade de rede de apoio social e da espiritualidade}

Tornou-se possível analisar nesta categoria os efeitos positivos que o apoio social e a espiritualidade poderão acrescentar na melhora do bem-estar dos cuidadores principais mediante abordagem paliativa, mostrando que revezar os cuidados com outros membros da família ${ }^{22}$, bem como, estimular à dimensão espiritual do cuidador são necessários para amenizar o estresse emocional causado e os permitem passar por esse processo de maneira menos dolorosa, 
proporcionando conforto e melhor aceitação ${ }^{28}$. Revelando por meio dos discursos de sentimentos de abandono, de sobrecarga, de exaustão emocional, além de ressaltar as afecções físicas que os limitam:

[...] Eu iria me sentir valorizada se os outros familiares ajudassem, fossem visitá-lo, ver o que estamos precisando, se está tudo ok, se está faltando alguma coisa, e isso eles não perguntam. Iria amenizar um pouco [a sobrecarga e cuidados] para mim e para minha mãe. (B1)

[...] Ninguém diz assim "vai em casa descansar que eu fico aqui com fulano, pelo menos uns três dias aqui pra tu" ninguém diz isso... Eu me sentiria melhor se recebesse ajuda, não ficaria tão sobrecarregada. (B3)

[...] É muito difícil ficar aqui presa dentro do hospital direto, não ter com quem trocar. [...] se tivesse outra pessoa para dividir comigo seria bem melhor, para resolver meus problemas da vida, espairecer um pouco [...] porque aqui no hospital, não é fácil não... (B4)

Iria melhorar para mim se eu tivesse alguém que pudesse dividir comigo, iria dividir um peso com outra pessoa, eu me sinto um pouco sufocada. (B6)

Precisei deixar minha vida pessoal e meus afazeres de lado no momento. Eu sinto falta de alguém que venha e fale: olhe, eu estou aqui com você, vai dar tudo certo, eu vou te ajudar, fique em casa, descansa um pouco... (B7)

[...] Eu acho que a família deveria ter mais disponibilidade em ajudar, passar alguns dias aqui para que eu pudesse ir em casa, revezar... e não jogasse tudo [responsabilidade do cuidado] em cima de mim. Então eu me sentiria menos sobrecarregada. (B8)

Além de enfatizarem o apoio social, os cuidadores também citaram a fé como suporte de conforto que atribui sentido ao cuidado, em que se busca sentido para a vida, mediante uma relação pessoal, coletiva e divina ${ }^{29}$, comportando-se como método de enfrentamento de seu sofrimento ${ }^{30}$ :

É Deus quem vai me dar mais forças ainda para eu cuidar do meu filho, ou ele levantando da cama ou não... é preciso ter muita fé e muito amor no coração. (B3)

A medicina tá ai, mas tem coisas que só Deus pode fazer. (B5)

Eu tenho fé em Deus que minha mãe vai se recuperar. (B10)

Acrescenta-se ainda que, a possibilidade de espaços de diálogos sobre Deus e espiritualidade e oração estabelece sensação de paz, saúde, felicidade e proteção, além disso, oferta compreensão e coerência em situações de doença e morte ${ }^{31-34}$

\section{Dificuldades na adaptação do cuidador aos cuidados paliativos na instituição hospitalar}

O isolamento social, a falta de socialização com outras pessoas e a falta de atividades não ligadas ao cuidado favorecem o surgimento de sintomas depressivos entre os cuidadores ${ }^{35}$. Desta forma, é necessário enfatizar a institucionalização do cuidador, no que se refere a sua inserção na rotina do familiar em CP. Estas abordagens descrevem-se nos seguintes relatos:

Não é fácil dormir numa poltrona, passar dias e noites assim, não é fácil. Para melhorar essa situação só quando ele vim pra casa né. (B1)

[...] Eu não me alimento bem [...] às vezes eu durmo sem jantar, porque ainda não me adaptei. Dormir em cadeira não é muito confortável [...] eu gostaria muito de trazer ele para casa, porque ele em casa eu estaria cuidando dele e cuidando de mim também. (B3)

O emocional fica muito mexido, não é nem de estar tomando conta dele aqui nem em casa, é de estar dentro de um hospital [...] Aqui você vê muitas situações de outros pacientes, e você não pode fazer nada... (B4)

[...] Apesar do hospital querer dar o máximo de conforto para a gente, ainda sim a cadeira é muito desconfortável. [...] Você ficar 24 h lá no hospital, chega um momento que você já está estressada, irritada, com as luzes, com tudo (B7)

Eu me sinto um pouco triste em estar dentro de um hospital. (B9)

É possível observar também, o reforço quanto às premissas do cuidado domiciliar nos $\mathrm{CP}$, comumente indicado para atenção às necessidades de pessoas com doenças crônicas e dependentes ${ }^{36}$, exigindo reorganização familiar para viabilizar a inserção de outras pessoas da família neste processo ${ }^{37}$, como possibilidade de amenizar estes problemas para o cuidador, tornando mais flexíveis algumas de suas atividades. Além disso, colabora para socialização do cuidador que permite o desenvolvimento na diversidade de papéis, alteração e equilíbrio de poder, conjunção de visões, contato físico, assim como, respeito mútuo ${ }^{38}$.

A pesquisa presta contribuição científica para profissionais de saúde e de enfermagem, influenciando para o planejamento e implementação de estratégias de prevenção do estresse emocional inserindo o cuidador no exercício assistencial paliativo, assim como, para o desenvolvimento de medidas de enfrentamento, por meio de práticas com 
abordagem paliativa baseadas em evidência científica que, especialmente no âmbito da enfermagem, constam as etapas da Sistematização da Assistência de Enfermagem.

Acrescenta-se ainda as limitações quanto ao cenário pandêmico atual, referente às medidas restritivas e realização de coleta de dados online, devido à indisponibilidade de internet e falta de habilidades com recursos digitais pelos participantes.

\section{CONSIDERAÇÕES FINAIS}

O estudo permitiu analisar o estresse emocional de cuidadores informais de pacientes em CP no âmbito hospitalar, a partir de aspectos relacionados ao cansaço físico e emocional, cuidado afetivo, à necessidade de rede de apoio social e espiritualidade, e adaptação aos CP institucionais.

Nessa perspectiva, notou-se a necessidade de o cuidador compreender seu papel social, buscar maneiras de fortalecer seu autocuidado e estimular suas fontes de enfrentamentos, seja por aspectos sociais, através de rotatividade de familiares a fim de segmentar as responsabilidades com as necessidades do familiar doente ou por aspectos espirituais. Para isso, é imprescindível que a equipe conheça as crenças espirituais dos cuidadores, promova espaços de diálogos com comunicação efetiva entre equipe, cuidadores e paciente, pois estas condições facilitam o processo de CP.

A escassez de estudos voltados para a temática revela a necessidade de mais pesquisas voltadas para o cuidador informal de pacientes em CP e de finitude de vida, buscando ampliar a multidimensionalidade do cuidado. Portanto, esta pesquisa possibilita a contribuição não só para profissionais, como também para as instituições de saúde e familiares.

\section{REFERÊNCIAS}

1. National Institute of Nursing Research. Palliative care: the relief you need when you have a serious illness. [internet] Bethesda: National Institute of Nursing Research; 2020 [cited 2021 Jun 26]. 01 p. Available from: https://www.ninr.nih.gov/sites/files/docs/palliative-care-brochure.pdf.

2. Radbruch L, Lima L de L, Kanaul F, Woodruff R, Yong J, Pastrana $T$, et al. Redefining palliative care - a new consensus-based definition. J Pain Symptom Manage. 2020 [cited 2021 Jun 28]; 60(4):754-64. DOI: https://doi.org/10.1016/j.jpainsymman.2020.04.027.

3. Silva RS, Oliveira ES, de Oliveira JF, Medeiros MO, Meira MV, Marinho CL. Family / caregiver's perspective on chronic pain in palliative care patients. Rev. Enfermería Actual [Internet]. 2020 [cited 2021 Mai 16]. DOI: http://dx.doi.org/10.15517/revenf.v0i38.37086.

4. Oliveira MB, de Souza NR, Bushatsky M, Dâmaso BF, Bezerra DM, de Brito JA. Oncological homecare: family and caregiver perception of palliative care. Esc. Anna Nery Rev. Enferm. [Internet]. 2017 [cited 2020 Out 25]; 21(2) e20170030. Available from: https://www.scielo.br/j/ean/a/p3fHvKrQS6ZzRNsPzRdB3gs/?lang=en.

5. Barbosa LM, Noronha K, Spyrides MH, Araújo CA. Qualidade de vida relacionada à saúde dos cuidadores formais de idosos institucionalizados em Natal. Rev. Bras. Est. Pop. [Internet]. 2017 [2020 Nov 26]; 34(2):391-414. DOI: https://doi.org/10.20947/S0102-3098a0004.

6. Rezende M, Costa SM. Impact of oncologic disease on relatives: psychopathological burden and symptomatology, relation and implications. Rev. invest. inov. saúde. [Internet]. 2018 [cited 2020 Nov 29]; 1(1):45-56. Available from: http://riis.essnortecvp.pt/index.php/RIIS/article/view/28/17.

7. Garassini MH. Los cuidadores de pacientes con cáncer: aprendizajes y recomendaciones a las familias, sociedades y centros de salud. Psicología y Salud [Internet]. 2016 [cited 2021 Jun 02]; 26(2):161-75. Available from: https://psicologiaysalud.uv.mx/index.php/psicysalud/article/view/2192/3919.

8. Bezerra BC, Oliveira SG, Dias LV. The caregiver in the hospital environment: an integrating review. Rev. urug. enferm. [Internet]. 2020 [cited 2020 Nov 25]; 15(1):1-17. Available from: http://rue.fenf.edu.uy/index.php/rue/article/view/288/305.

9. Santos WP, de Freitas FB, de Sousa VA, Oliveira AM, Santos JM, Gouveia BL. Overburden on elderly caregivers who care for dependent elderl. Rev. Cuid. (Bucaramanga. 2010). [Internet]. 2019 [cited 2020 Nov 28]; 10(2):e607. DOI: https://doi.org/10.15649/cuidarte.v10i2.607.

10. Pessalacia JD, Silva AE, Araújo AH, de Lacerda MA, dos Santos KC. Experiences of carevigers in the palliative care and support networks. Rev. enferm. UFPE on line [Internet]. 2018 [cited 2020 Out 23]; 12(11):2916-22. DOI: https://doi.org/10.5205/19818963-v12i11a236208p2916-2922-2018.

11. Araújo AS, Pedroso TG. The relationship between expressed emotion and sociodemographic variables, early stress and stress symptoms in informal caregivers of people with mental disorders. Cad. Bras. Ter. Ocup. [Internet]. 2019 [cited 2020 Nov 30]; 27(4):743-53. DOI: http://doi.org/10.4322/2526-8910.ctoAO1843. 
12. Gutierrez DM, Sousa GS, Figueiredo AE, Ribeiro MN, Diniz CX, Nobre GA. Subjective life experiences of Family caregivers of dependent older adults. Ciênc. Saúde Colet. [Internet]. 2021 [cited 2021 Jun 17]; 26(1):47-56. DOI: https://doi.org/10.1590/1413-81232020261.30402020.

13. Litzelman K, Kent EE, Rowland JH. Social factors in informal cancer caregivers: the interrelationships among social stressors, relationship quality, and family functioning in the CanCORS data set. Cancer [Internet]. 2016 [cited 2021 Jun 02]; 122(2):278-86. DOI: https://doi.org/10.1002/cncr.29741.

14. Pavarini SC, Bregola AG, Luchesi BR, Oliveira D, Orlandi FS, Moura FG et al. Social and health-related predictors of Family function in older spousal caregivers: a cross-sectional study. Dement. Neuropsychol. [Internet]. 2020 [cited 2021 Jun 12]; 14(4):372-8. DOI: http://dx.doi.org/10.1590/1980-57642020dn14-040007.

15. Rigoni L, Bruhn RF, de Cicco R, Kanda JL, Matos LL. Quality of life impairment in patients with head and neck cancer and their caregivers: a comparative study. Braz. j. otorhinolaryngol. [Internet]. 2016 [cited 2021 Jun 12]; 82(6):680-6. DOI: https://doi.org/10.1016/j.bjorl.2015.12.012.

16. Tong A, Sainsbury P, Craig J. Consolidated criteria for reporting qualitative research (COREQ): a 32-item checklist for interviews and focus groups. Int. j. qual. health care. [Internet]. 2007 [cited 2021 Apr 23]; 19(6):349-57. DOI: http://doi.org/10.1093/intqhc/mzm042.

17. Minayo MCS. Amostragem e saturação em pesquisa qualitativa: consensos e controvérsias. Rev. Pesqui. Quali [Internet]. 2017 [cited 2021 Mai 28]; 5(7):1-12. Available from: http://editora.sepq.org.br/rpq/article/view/82.

18. Bardin L. Análise de Conteúdo. 1. ed. São Paulo: Edições 70; 2016.

19. Brasil. Lei $n=13.709$, de 14 de agosto de 2018. Dispõe sobre a proteção de dados pessoais e altera a Lei $n=12.965$, de 23 de abril de 2014. Diário Oficial da União. 2018 Aug 15;155 (157 seção 1):59-64. Available from: http://www.planalto.gov.br/ccivil_03/_ato2015-2018/2018/lei/l13709.htm.

20. Brasil. Ofício Circular no 2/2021/CONEP/SECNS/MS. Orienta pesquisadores e Comitês de Ética em Pesquisa em relação a procedimentos que envolvam o contato com participantes e/ou coleta de dados em qualquer etapa da pesquisa, em ambiente virtual. Comissão Nacional de Ética em Pesquisa. 2021 Feb 24. Available from: http://conselho.saude.gov.br/images/Oficio_Circular_2_24fev2021.pdf.

21. Lima CP, Machado MA. Main Caregivers Facing Death Experience and Its Meanings. Psicol. ciênc. prof. [Internet]. 2018 [cited 2021 Apr 15]; 38(1):88-101. DOI: http://doi.org/10.1590/1982-3703002642015.

22. Lima LE, Santana ME, Correa Júnior AJS, Vasconcelos EV. Together we resist, separated we fall out: experiences of family caregivers of cancer patients in palliative care. Rev. Fun. Care Online [Internet]. 2019 [cited 2021 Mai 16]; 11(4):931-6. DOI: https://doi.org/10.9789/2175-5361.2019.v11i4.931-936.

23. Kobayasi DY, Rodrigues RA, Fhon JR, Silva LM, Souza AC, Chayamiti EM. Overload, social support network and emotional stress on the caregiver of elderly. Av. enferm. [Internet]. 2019 [cited 2020 Nov 30]; 37(2):140-8. DOI: http://doi.org/10.15446/av.enferm.v37n2.73044.

24. Cámara SM, Moralb PA, Fernándeza LM, Osunab AF, Casadob RP. Problemas en el proceso de adaptación a los câmbios en personas cuidadoras familiares de mayores con demencia. Gac. Sanit. [Internet]. 2016 [cited 2021 Mai 28]; 30(3):201-7. DOI: https://dx.doi.org/10.1016/j.gaceta.2016.02.004.

25. Couto AM, Caldas CP, Castro EA. Family caregiver of older adults and Cultural Care in Nursing care. Rev. bras. enferm. [Internet]. 2018 [cited 2021 Mai 28]; 71(3):959-66. DOI: https://doi.org/10.1590/0034-7167-2017-0105.

26. Mendez-Luck CA, Anthony KP, Guerrero LR. Burden and bad days among mexican-origin women caregivers. J. Gerontol. Ser. B, Psychol sci. soc. sci. [Internet]. 2019 [cited 2021 Mai 28]; 75(8):1719- 30. DOI: http://doi.org/10.1093/geronb/gbz102.

27. Mattos EB, Oliveira JP, Novelli MM. The demands of care and self-care from the family caregiver's of the elderly with dementia perspective. Rev. bras. geriatr. gerontol. [Internet]. 2020 [cited 2021 Apr 29]; 23(3). DOI: https://doi.org/10.1590/198122562020023.200189

28. Maffei B, Arrieira IC, Ferreira RA, Cardoso DH. Coping strategies of caregivers of patients receiving palliative home care. Psicol. teor. e prát. [Internet]. 2019 [cited 2021 May 28]; 21(3):303.22. DOI: http://dx.doi.org/10.5935/19806906/psicologia.v21n3p303-322.

29. Evangelista CB, Lopes ME, Costa SF, Abrão FM, Batista PS, Oliveira RC. Spirituality in patient care under palliative care: A study with nurses. Esc. Anna Nery Rev. Enferm. [Internet]. 2016 [cited 2021 May 28]; 20(1):176-82. DOI: https://doi.org/10.5935/1414-8145.20160023.

30. Arrieira IC, Thofehrn MB, Milbrath VM, Schwonke CR, Cardoso DH, Fripp JC. The meaning of spirituality in the transience of life. Esc. Anna Nery Rev. Enferm. [Internet]. 2017 [cited 2021 Jun 25]; 21(1). Available from: https://www.scielo.br/j/ean/a/F5n46JqtVcqbG8PvW68FqML/?lang=en.

31. Arrieira IC, Thoferhn MB, Schaefer OM, Fonseca AD, Kantorski LP, Cardoso DH. The sense of spiritual care in the integrality of attention in palliative care. Rev. gaúch. enferm. [Internet]. 2017 [cited 2021 May 28]; 38(3):e58737. DOI: http://doi.org/10.1590/1983-1447.2017.03.58737. 
32. Kristanti MS, Effendy C, Utarini A, Vernooij-Dassen M, Engels Y. The experience of family caregiversof patients with cancer in an Asian country: A grounded theory approach. Palliat. med. [Internet]. 2019 [cited 2021 May 28]; 33(6):676-84, 2019. DOI: http://doi.org/10.1177/0269216319833260.

33. Miqueletto M, Silva L, Figueira CB, dos Santos MR, Szylit R, Ichikawa CRF. Espiritualidade de famílias com um ente querido em situação de final de vida. Rev. Cuid. [Internet]. 2017 [cited 2021 May 28]; 8(2):1616-27. DOI: http://dx.doi.org/10.15649/cuidarte.v8i2.391.

34. Queiroz AC, da Silva MP, Dantas MC, Fonseca AC, de Brito DT, Agra G. Care aimed at family members of people in human finitude. Res. Soc. Dev. [Internet]. 2021 [cited 2021 Jun 12]; 10(2):e7310212151. DOI: http://doi.org/10.33448/rsd-v10i2.12151.

35. Lacerda MS, Prado PR, Barros AL, Lopes JL. Depressive symptoms in the family caregivers of patients with heart failure: an integrative review. Rev. gaúch. enferm. [Internet]. 2019 [cited 2021 May 16]; 40:e20180057. DOI: http://doi.org/10.1590/19831447.2019.20180057.

36. Simião VM, Mioto RC. The palliative care and household in country of Latin America. Saúde debate [Internet]. 2016 [cited 2021 Jun 09]; 40(108):156-69. DOI: http://doi.org/10.1590/0103-1104-20161080013.

37. Fernandes CS, Angelo M. Family caregivers: what do they need? An integrative review. Rev. Esc. Enferm. USP. [Internet]. 2016 [cited 2021 May 30]; 50(4):675-82. DOI: https://doi.org/10.1590/S0080-623420160000500019.

38. Cardoso AC, Noguez PT, Oliveira SG, Porto AR, Perboni JS, Farias TA. Rede de apoio e sustentação dos cuidadores familiares de pacientes em cuidados paliativos no domicílio. Enferm. foco. 2019 [cited 2021 Jun 09]; 10(3):70-5. Available from: http://revista.cofen.gov.br/index.php/enfermagem/article/view/1792/579. 